\title{
Characteristics of prescriptions and costs for acute upper respiratory tract infections in Chinese outpatient pediatric patients: a nationwide cross- sectional study
}

\section{Shan Wang}

Chinese PLA General Hospital

Lihua Liu

Chinese PLA General Hospital

Jianchao Liu

Chinese PLA General Hospital

Likun Miao

Chinese PLA General Hospital

Qian Zhuang

Chinese PLA General Hospital

Ning Guo

Massachusetts General Hospital

Jing Zhao

Chinese PLA General Hospital

Quanzheng Li

Massachusetts General Hospital

Guoquan Ren ( $\nabla$ renguoquan301@outlook.com )

Chinese PLA General Hospital https://orcid.org/0000-0001-5041-6811

Research article

Keywords: upper respiratory tract infections, prescription, cost, pediatric, outpatient, China

Posted Date: February 11th, 2020

DOI: https://doi.org/10.21203/rs.2.23136/v1

License: (c) (1) This work is licensed under a Creative Commons Attribution 4.0 International License. Read Full License 
Version of Record: A version of this preprint was published at BMC Complementary Medicine and Therapies on November 16th, 2020. See the published version at https://doi.org/10.1186/s12906-020-03141-w. 


\section{Abstract}

Background

To understand the characteristics of prescriptions and costs in pediatric patients with acute upper respiratory infection (AURI) is important for the regulation of outpatient care and reimbursement policy. This study aims to provide evidence on these issues that was in short supply.

Methods

We conducted a retrospective cross-sectional study based on data from 138 hospitals across the country. All pediatric patients aged 0-14 years diagnosed with uncomplicated AURI visiting hospital ambulatory departments from 1 January 2015 to 31 December 2017 were included. We reported characteristics of pediatric patients diagnosed with AURI, the average number of medications prescribed per encounter, the categories of medication used and their percentages, the cost per visit and prescription costs of drugs. For these measurements, discrepancies among diverse groups of age, regions, insurance types, and AURI categories were compared. Kruskal-Wallis nonparametric test and Student-Newman-Keuls test were performed to test differences among subgroups.

Results

A total of 1,002,687 clinical records with 2,682,118 prescriptions were collected and analyzed. The average number of drugs prescribed per encounter was 2.8 . The most frequently prescribed medication was Chinese traditional patent medicines (CTPM) (36.5\% of overall prescriptions) followed by antibiotics $(18.1 \%)$. It showed a preference of CPTM over western drugs. The median cost per visit was 119.97 CNY. The median drug cost per visit was 92.70 CNY. The expenditures of antibiotics and CTPM per visit (40.54CNY and 39.35CNY) were among the three highest categories of drugs. The percentage of out-of-pocket patients reached $65.9 \%$. Disparities have been showed among subgroups of different ages, regions, and insurance types.

\section{Conclusions}

The high volume of CPTM usage is the typical feature in outpatient care of AURI pediatric patients in China. The rational and cost-effective use of CPTM and antibiotics still faces challenges. The reimbursement for child AURI cases needs to be enhanced.

\section{Background}

Pediatric outpatient visits took up $9.8 \%$ of total outpatient visits in China, among which acute upper respiratory tract infection (AURI) is the first common cause of sicknesses, accounting for more than $60 \%$ of total visits [1]. The primary strategy for the management of AURI is medication therapy. As children are highly vulnerable to irrational drug use, the study of prescriptions in children with AURI are of great importance. There are also financial concerns for the pediatric outpatient cases in consideration of its high incidence and vast price gaps in pharmaceutical market. Evidence-based cost analysis is also essential for 
payment and reimbursement policy. However, the available research for prescription patterns and its relevant spending in Chinese pediatric population with AURI remained in short supply. The existing published studies were usually limited to particular facilities or regions, selected insufficient samples, or non-English language [2-13].Studies using nationwide sample has not been found yet. The results also appear large inconsistency. The insufficient evidence makes it harder to monitor and regulate outpatient services resulting in the lag of policy adjustment [14-16].

In the present study, we aim to implement a comprehensive investigation in characteristics of prescriptions and spending of pediatric patients diagnosed with AURI in outpatient sectors. We obtain a large sample of outpatient cases with individual-level clinical information covering a considerable portion of public hospitals across the country. The study tends to supply a reliable evidence on this issue. Also, it is the first attempt to use patient-level information in such scale to analyze pediatric outpatient care in China.

\section{Methods}

\section{Study design and data source}

We conducted a retrospective cross-sectional study of pediatric patients diagnosed with uncomplicated AURI who visited hospital ambulatory departments, including emergency departments from 1 January 2015 to 31 December 2017. 138 public tertiary hospitals from 111 cities in 31 provinces were selected. These hospitals apply the identical hospital information system so that their clinical records could be aggregated directly without technical barriers. The information directly extracted from hospital information system. All the records were de-identified before analysis so that confidentiality was assured.

\section{Patients Selection And Data Processing}

We include all pediatric patients aged 0-14 years. Patients were identified according to J00-J06 code section representing all types of AURI diagnoses in the 10th revision of the International Statistical Classification of Diseases and Related Health Problems (ICD-10, Chinese 2011 edition)[17]. For those diagnoses without code in the records, we employed the Natural Language Processing (NLP) technique [18] to standardize the information to identify the AURI patients. The original diagnosis entries were distinguished via the keywords and automatically classified and standardized once matched. The prescriptions also went through the process of standardization by NLP. Original names of medications on prescriptions were distinguished based on China Pharmacopeia (2015 edition) [19].

\section{Data analysis}

In our analysis, patients were divided into four age groups as $\leq 28$ days, 29 days to less than 1 year, 1-4 years, and 5-14 years. Hospitals were sorted into four economic regions, namely Northeast, East, Central, and West region, according to the National Bureau of Statistics [20]. We also classified four consultation types in terms of the physician's professional title and the department where consultations take place. If a physician with a senior professional title provided consultation in the ambulatory department or emergency 
department, we categorized the case into "expert consultation (EC)" or "expert consultation in the emergency department (EDEC)", respectively. Otherwise, we sorted the consultation into "general consultation (GC)" or "general consultation in the emergency department (EDGC)". Health insurances of patients were classified into seven groups including Urban Resident Basic Medical Insurance (URBMI), New Rural Cooperative Medical System (NRCMS), Commercial Health Insurance (CHI), Welfare Health Care (WHC), Preferential Health Care (PHC), Out-of-pocket Payment (OOP) and Others. URBMI and NRCMS are two of the three national fundamental social medical insurance schemes in China [21]. $\mathrm{CHI}$ includes all kinds of commercial health insurances supplemented to the social health insurances. WHC refers to the healthcare welfare, which is mostly provided to national civil servants and staff of large state-owned enterprises at the stage. PHC beneficiaries are usually dependents of military personnel and other prior groups who meet the conditions that can enjoy exclusive medical benefits decided by the hospitals. OOP means the patients pay by themselves. "Others" refers to the remaining situations.

We performed a descriptive analysis using SAS software, Version 9.4 (SAS Institute Inc., North Carolina, USA). We reported characteristics of pediatric patients diagnosed with AURI, the average number of medications prescribed per encounter, the categories of medication used and their percentages, the cost per visit and prescription costs of different category of drugs. For these measurements, we compared differences among different age bands, regions, insurance types, and AURI categories.

In our calculation, one encounter stands for an event of a particular patient visiting a specific hospital on a particular day. Multiple visits of the same patient in the same hospital within seven days counted as one encounter according to the typical duration of AURI. The expenditure per case was calculated by adding up the charges of each item based on fee-for-service payment.

We employed Kruskal-Wallis nonparametric test to evaluate differences among subgroups. StudentNewman-Keuls test was used to do stepwise multiple comparisons.

\section{Results}

\section{Characteristics of pediatric patients diagnosed with AURI}

Between Jan 1, 2015, and Dec 31, 2017, the total outpatient visits of children diagnosed with AURI without comorbidities and complexities was 1,002,687 in studied hospitals. Acute tonsillitis was the most frequent diagnosis other than unspecified AURI. The volume of visits each year remains stable, with less than $10 \%$ fluctuate (Table 1). 
Table 1

Characteristics of outpatient visits of pediatric AURI patients in 2015-2017

\begin{tabular}{|c|c|c|c|c|c|c|c|c|}
\hline \multirow{2}{*}{ Characteristics } & \multicolumn{2}{|l|}{ Total } & \multicolumn{2}{|l|}{2015} & \multicolumn{2}{|l|}{2016} & \multicolumn{2}{|l|}{2017} \\
\hline & \multicolumn{2}{|c|}{$(N=1002687)$} & \multicolumn{2}{|c|}{$(\mathrm{N}=326617)$} & \multicolumn{2}{|c|}{$(N=357366)$} & \multicolumn{2}{|c|}{$(N=318704)$} \\
\hline \multicolumn{9}{|l|}{$\operatorname{Sex}(n, \%)$} \\
\hline Male & 556797 & $(55 \cdot 5 \%)$ & 182885 & $(56 \cdot 0 \%)$ & 198389 & $(55 \cdot 5 \%)$ & 175523 & $(55 \cdot 1 \%)$ \\
\hline Female & 445890 & $(44 \cdot 5 \%)$ & 143732 & $(44 \cdot 0 \%)$ & 158977 & $(44 \cdot 5 \%)$ & 143181 & $(44.9 \%)$ \\
\hline \multicolumn{9}{|l|}{ Age $(n, \%)$} \\
\hline$\leq 28$ days & 1503 & $(0 \cdot 1 \%)$ & 469 & $(0 \cdot 1 \%)$ & 575 & $(0 \cdot 2 \%)$ & 459 & $(0 \cdot 1 \%)$ \\
\hline $\begin{array}{l}>28 \text { days and < } \\
1 \text { year }\end{array}$ & 78274 & $(7 \cdot 8 \%)$ & 29428 & $(9 \cdot 0 \%)$ & 24848 & $(7 \cdot 0 \%)$ & 23998 & $(7 \cdot 5 \%)$ \\
\hline $1-4$ years old & 509471 & $(50 \cdot 8 \%)$ & 172800 & $(52 \cdot 9 \%)$ & 182607 & $(51 \cdot 1 \%)$ & 154064 & $(48 \cdot 3 \%)$ \\
\hline $5-14$ years old & 413439 & $(41 \cdot 2 \%)$ & 123920 & $(37 \cdot 9 \%)$ & 149336 & $(41 \cdot 8 \%)$ & 140183 & $(44 \cdot 0 \%)$ \\
\hline \multicolumn{9}{|l|}{$\begin{array}{l}\text { Economic } \\
\text { region }(n, \%)\end{array}$} \\
\hline Northeast & 115570 & $(11 \cdot 5 \%)$ & 38762 & $(11 \cdot 9 \%)$ & 43132 & $(12 \cdot 1 \%)$ & 33676 & $(10 \cdot 6 \%)$ \\
\hline East & 618017 & $(61 \cdot 6 \%)$ & 202141 & $(61 \cdot 9 \%)$ & 225812 & $(63 \cdot 2 \%)$ & 190064 & $(59 \cdot 6 \%)$ \\
\hline Central & 106459 & $(10 \cdot 6 \%)$ & 37065 & $(11 \cdot 3 \%)$ & 35981 & $(10 \cdot 1 \%)$ & 33413 & $(10 \cdot 5 \%)$ \\
\hline West & 162641 & $(16 \cdot 2 \%)$ & 48649 & $(14 \cdot 9 \%)$ & 52441 & $(14 \cdot 7 \%)$ & 61551 & $(19 \cdot 3 \%)$ \\
\hline \multicolumn{9}{|l|}{$\begin{array}{l}\text { Type of health } \\
\text { insurance or } \\
\text { health care plan } \\
(n, \%)\end{array}$} \\
\hline URBMI & 178917 & $(17 \cdot 8 \%)$ & 49019 & $(15 \cdot 0 \%)$ & 64993 & $(18 \cdot 2 \%)$ & 64905 & $(20 \cdot 4 \%)$ \\
\hline NRCMS & 5274 & $(0.5 \%)$ & 1501 & $(0.5 \%)$ & 1978 & $(0 \cdot 6 \%)$ & 1795 & $(0 \cdot 6 \%)$ \\
\hline $\mathrm{CHI}$ & 472 & $(0.0 \%)$ & 193 & $(0 \cdot 1 \%)$ & 146 & $(0.0 \%)$ & 133 & $(0.0 \%)$ \\
\hline WHC & 6458 & $(0 \cdot 6 \%)$ & 2397 & $(0 \cdot 7 \%)$ & 2298 & $(0 \cdot 6 \%)$ & 1763 & $(0 \cdot 6 \%)$ \\
\hline PHC & 141413 & $(14 \cdot 1 \%)$ & 40030 & $(12 \cdot 3 \%)$ & 46172 & $(12.9 \%)$ & 55211 & $(17 \cdot 3 \%)$ \\
\hline OOP & 660292 & $(65.9 \%)$ & 229749 & $(70 \cdot 3 \%)$ & 238004 & $(66 \cdot 6 \%)$ & 192539 & $(60 \cdot 4 \%)$ \\
\hline
\end{tabular}

* This group comprised the patients with the diagnosis of "acute laryngitis and tracheitis(J04)" and "acute obstructive laryngitis and epiglottitis (J05)".

URBMI = Urban Resident Basic Medical Insurance. NRCMS = New Rural Cooperative Medical System. $\mathrm{CHI}$ $=$ Commercial Health Insurance. WHC $=$ Welfare Health Care. PHC $=$ Preferential Health Care. OOP = Out of-pocket Payment. ED = Emergency department. ARUI = Acute Respiratory Upper Tract Infections. 


\begin{tabular}{|c|c|c|c|c|c|c|c|c|}
\hline \multirow{3}{*}{$\begin{array}{l}\text { Characteristics } \\
\text { Others }\end{array}$} & \multirow{2}{*}{\multicolumn{2}{|c|}{$\begin{array}{l}\text { Total } \\
(N=1002687)\end{array}$}} & \multirow{2}{*}{\multicolumn{2}{|c|}{$\begin{array}{l}2015 \\
(N=326617)\end{array}$}} & \multirow{2}{*}{\multicolumn{2}{|c|}{$\begin{array}{l}2016 \\
(N=357366)\end{array}$}} & \multirow{2}{*}{\multicolumn{2}{|c|}{$\begin{array}{l}2017 \\
(N=318704)\end{array}$}} \\
\hline & & & & & & & & \\
\hline & 9861 & $(1 \cdot 0 \%)$ & 3728 & $(1 \cdot 1 \%)$ & 3775 & $(1 \cdot 1 \%)$ & 2358 & $(0 \cdot 7 \%)$ \\
\hline \multicolumn{9}{|l|}{$\begin{array}{l}\text { Type of the } \\
\text { consultation ( } \\
\text { \%) }\end{array}$} \\
\hline $\begin{array}{l}\text { General } \\
\text { consultation }\end{array}$ & 337423 & $(33 \cdot 7 \%)$ & 109376 & $(33 \cdot 5 \%)$ & 119134 & $(33 \cdot 3 \%)$ & 108913 & $(34.2 \%)$ \\
\hline $\begin{array}{l}\text { Expert } \\
\text { consultation }\end{array}$ & 228325 & $(22 \cdot 8 \%)$ & 80719 & $(24 \cdot 7 \%)$ & 83961 & $(23 \cdot 5 \%)$ & 63645 & $(20 \cdot 0 \%)$ \\
\hline $\begin{array}{l}\text { General } \\
\text { consultation in } \\
\text { ED }\end{array}$ & 367579 & $(36 \cdot 7 \%)$ & 115947 & $(35 \cdot 5 \%)$ & 128739 & $(36 \cdot 0 \%)$ & 122893 & $(38.6 \%)$ \\
\hline $\begin{array}{l}\text { Expert } \\
\text { consultation in } \\
\text { ED }\end{array}$ & 69360 & $(6 \cdot 9 \%)$ & 20575 & $(6 \cdot 3 \%)$ & 25532 & $(7 \cdot 1 \%)$ & 23253 & $(7 \cdot 3 \%)$ \\
\hline \multicolumn{9}{|l|}{$\begin{array}{l}\text { Classification } \\
\text { of AURI }(n, \%)\end{array}$} \\
\hline $\begin{array}{l}\text { Acute } \\
\text { nasopharyngitis }\end{array}$ & 1094 & $(0 \cdot 1 \%)$ & 268 & $(0 \cdot 1 \%)$ & 452 & $(0 \cdot 1 \%)$ & 374 & $(0 \cdot 1 \%)$ \\
\hline Acute sinusitis & 3867 & $(0 \cdot 4 \%)$ & 1213 & $(0 \cdot 4 \%)$ & 1262 & $(0 \cdot 4 \%)$ & 1392 & $(0 \cdot 4 \%)$ \\
\hline $\begin{array}{l}\text { Acute } \\
\text { pharyngitis }\end{array}$ & 57775 & $(5 \cdot 8 \%)$ & 18331 & $(5 \cdot 6 \%)$ & 19875 & $(5 \cdot \%)$ & 19569 & $(6 \cdot 1 \%)$ \\
\hline Acute tonsillitis & 137128 & $(13 \cdot 7 \%)$ & 40537 & $(12 \cdot 4 \%)$ & 48642 & $(13 \cdot 6 \%)$ & 47949 & $(15 \cdot 0 \%)$ \\
\hline $\begin{array}{l}\text { Acute laryngitis, } \\
\text { tracheitis \& } \\
\text { epiglottitis * }\end{array}$ & 70919 & $(7 \cdot 1 \%)$ & 22489 & $(6 \cdot 9 \%)$ & 25707 & $(7 \cdot 2 \%)$ & 22723 & $(7 \cdot 1 \%)$ \\
\hline $\begin{array}{l}\text { Unspecified } \\
\text { AURI }\end{array}$ & 731904 & $(73 \cdot 0 \%)$ & 243779 & $(74 \cdot 6 \%)$ & 261428 & $(73 \cdot 2 \%)$ & 226697 & $(71 \cdot 1 \%)$ \\
\hline \multicolumn{9}{|c|}{$\begin{array}{l}\text { * This group comprised the patients with the diagnosis of "acute laryngitis and tracheitis(J04)" and } \\
\text { "acute obstructive laryngitis and epiglottitis (J05)". }\end{array}$} \\
\hline \multicolumn{9}{|c|}{$\begin{array}{l}\text { URBMI = Urban Resident Basic Medical Insurance. NRCMS }=\text { New Rural Cooperative Medical System. CHI } \\
=\text { Commercial Health Insurance. WHC }=\text { Welfare Health Care. PHC = Preferential Health Care. OOP = Out- } \\
\text { of-pocket Payment. ED = Emergency department. ARUI = Acute Respiratory Upper Tract Infections. }\end{array}$} \\
\hline
\end{tabular}

\section{Quantity Of Prescribed Medications}

A total of 2774 classes of medications have been prescribed 2,682,118 times among 938,687 out of $1,002,687$ visits. These medications were classified into 146 subcategories of 27 major categories according 
to the China Pharmacopeia (2015 edition) [11]. The average number of drugs prescribed per visit was 2.8 . The most typical situation was prescribing two classes of medications (27.3\%) per visit, followed by three (23.9\%) and one (21.7\%).

The average number of medications prescribed per visit increased in all subgroups from 2015 to 2017. Distinct differences were shown among different age bands, regions, insurance types, and AURI categories ( $p<0.0001$ for all tests), while little distances were observed among various consultation types. The younger the child was, the fewer the quantity of medication was prescribed, where $48.5 \%$ of newborns were prescribed only one medication per visit. The children in the western region received the highest supply of drugs per visit, while those in northeastern hospitals were prescribed with the lowest. There was no significant difference in numbers of medicines prescribed by experts, whether in the emergency department or not. $\mathrm{CHI}$ beneficiaries were averagely prescribed three drugs per encounter, which was notably higher than children with other insurance plans. In cases diagnosed with acute laryngitis, tracheitis \& epiglottitis, the most medications (around 3.3 classes) were prescribed. By comparison, acute sinusitis cases were offered the least medication preparations (Fig. 1).

\section{Categories Of Prescribed Medications}

The most frequently prescribed medication was Chinese traditional patent medicines (CTPM), the total amount of which reached $36.5 \%$ of overall prescriptions, followed by antibiotics (18.1\%) (Fig. 2). The percentage of visits during which children were prescribed CTPM rose from $63.6 \%$ in 2015 to $71.4 \%$ in 2017 , while the figures for antibiotics was from $40.4-46.2 \%$. Besides, $17.4 \%$ of cases were only prescribed CTPM in three years. In the northeast region, there were more children (around $30 \%$ ) only consuming CTPM.

\section{Prescription Diversity}

As illustrated in Fig. 3, we used one color to label the same major category of drugs and noted the share of its prescription volume as well. The colored squares were sorted by percentages from top to bottom.

Notably, the use of CTPM to treat AURI was widespread among children in China, regardless of the age, region, insurance type, and consultation type. It ranked the first in almost every subgroup. The top five major categories remained stable in each group. Still, disparities existed at the same time. For example, the antipyretic-analgesics medicines were used more often in children under one-year-old than elders. They were prescribed less frequently in the northeast region than in other areas, and they played a more significant role in the emergency departments.

Some subgroups showed their own typical features. For instance, the usage of traditional Chinese medicinal materials in all children groups was relatively small (between 0-3\%) except for PHC beneficiaries, which was up to $15.5 \%$. In another example, we could find that non-antibiotic anti-infective drugs prescribed by expert physicians in the emergency department were significantly higher than other consultant types. Among different AURI classification groups, the disparity of prescription was prominent. 
Five pairs of CTPM and western (chemical) drugs of high frequencies which had similar effects mainly in alleviating symptoms of AURI were chosen to be compared. In three out of five pairs, the prescription frequencies of CTPM were 2.1-8.6 times of those of western drugs. While in the other two pairs, however, the volumes of western drug prescriptions were a litter higher than Chinese patent medicines. And the median drug costs per encounter for Chinese patent drugs were higher than those for western drugs in all selected pairs (Table 2).

Table 2 Comparison of volume and costs between CPTM and western drugs with similar effects

\begin{tabular}{|c|c|c|c|c|}
\hline & Major Category & Subcategory & $\begin{array}{l}\text { Frequency of } \\
\text { prescription }\end{array}$ & $\begin{array}{l}\text { Median drug costs } \\
\text { per visit (CNY) }\end{array}$ \\
\hline \multirow[t]{2}{*}{1} & $\begin{array}{l}\text { Chinese traditional } \\
\text { patent medicines }\end{array}$ & Chinese heat-clearing formula & 340945 & 38.78 \\
\hline & $\begin{array}{l}\text { Antipyretic-analgesics } \\
\text { drugs }\end{array}$ & Analgesic-antipyretic drugs & 49716 & 35.27 \\
\hline \multirow[t]{2}{*}{2} & $\begin{array}{l}\text { Chinese traditional } \\
\text { patent medicines }\end{array}$ & $\begin{array}{l}\text { Chinese cough-suppressing } \\
\text { panting-calming formula }\end{array}$ & 158845 & 31.19 \\
\hline & $\begin{array}{l}\text { Respiratory system } \\
\text { drugs }\end{array}$ & Antitussives & 49848 & 23.48 \\
\hline \multirow[t]{2}{*}{3} & $\begin{array}{l}\text { Chinese traditional } \\
\text { patent medicines }\end{array}$ & $\begin{array}{l}\text { Chinese nasal congestion } \\
\text { relieving formula }\end{array}$ & 149133 & 66.08 \\
\hline & Specialist drugs & Otorhinolaryngology drugs & 17419 & 48.82 \\
\hline \multirow[t]{2}{*}{4} & $\begin{array}{l}\text { Chinese traditional } \\
\text { patent medicines }\end{array}$ & Chinese expectorant formula & 84742 & 25.99 \\
\hline & $\begin{array}{l}\text { Respiratory system } \\
\text { drugs }\end{array}$ & Expectorants & 107254 & 24.5 \\
\hline \multirow[t]{2}{*}{5} & $\begin{array}{l}\text { Chinese traditional } \\
\text { patent medicines }\end{array}$ & $\begin{array}{l}\text { Chinese traditional digestive } \\
\text { formula }\end{array}$ & 9160 & 45.57 \\
\hline & $\begin{array}{l}\text { Digestive system } \\
\text { drugs }\end{array}$ & $\begin{array}{l}\text { Stomachic and digestion aid } \\
\text { medicine }\end{array}$ & 10826 & 37.45 \\
\hline
\end{tabular}

\section{Costs Of Outpatient Care For Pediatric Patients With AURI}

On a per visit basis, the median cost was 119.97 CNY with an average annual growth rate of $5.5 \%$ between 2015 and 2017 (Fig. 4). Only two subgroups (acute nasopharyngitis and acute pharyngitis) showed a downward trend in the cost per visit when compared the year 2017 with 2015. The spending varied significantly among subgroups of different age, regions, insurance types, and AURI classifications $(P<$ 0.0001 in all tests). Specifically, the expense on elder children aged 5-14 years (135.50 CNY) is nearly twice as much as the expenses of neonates (69.05 CNY) and around 1.5 times of the that of children younger than one (88.00 CNY). Compared with the cases in central ( $99.07 \mathrm{CNY}$ ) and west regions ( $80.45 \mathrm{CNY})$, the 
expenses of those in the east (133.28 CNY) and northeast region (136.58 CNY) are much higher. CHI participants (194.83 CNY) experienced the highest cost, while the PHC beneficiaries spent the least (103.8 CNY). No significant difference was observed between the spending of URBMI (161.80 CNY) and WHC (166.06 CNY) beneficiaries as well as those of NRCMS (108.99 CNY) and OOP (113.10 CNY). However, the cost per visit of former two schemes was significantly higher than the latter ones. In terms of different classification of AURI, acute nasopharyngitis appeared to be the costliest type of disease (235.60 CNY), while unspecified AURI showed the least spending $(111 \cdot 41 \mathrm{CNY})$ which is less than the half of the former.

The costly cases that spent more than 520 CNY took up $4.0 \%$ of total consultations, with the highest cost of 984.85 CNY. Subgroups covered by $\mathrm{CHI}$ and diagnosed with acute sinusitis produced the highest proportions of expensive cases, which was $13.1 \%$ and $10.3 \%$, respectively.

The median drug cost per visit was $92.70 \mathrm{CNY}$. The five most costly drugs in one prescription were immunoregulation drugs (90.16 CNY), antibiotics (40.54 CNY), Chinese traditional patent medicines (39.35 $\mathrm{CNY}$ ), non-antibiotic anti-infective drugs (32.30 CNY), and specialty medicines (32.18 CNY). In contrast with CTPM, the median spending of Chinese herbal medicine per prescription was only 3.71CNY, being less than $10 \%$ of spending of CTPM.

\section{Discussion}

In our results, the average number of medications prescribed per encounter was similar with some studies $[22,23]$. It is noteworthy that CTPM stood out of all kinds of drugs, ranking the first in total prescription volume, covering more than two-thirds of the visits. And there was nearly 20 percent of cases only prescribed CTPM. It suggests a distinct feature of prescriptions in China, compared with results in foreign studies [2432]. For example, the most commonly prescribed medicines for AURI children in a Danish research were respiratory medicines (47.0\%), antimicrobials (30.7\%), and analgesic-antipyretics (18.8\%) [25], which in our study were only $9.0 \% 18.1 \%$, and $11.9 \%$, respectively, ranking below CTPM. Moreover, it showed that there was a notable preference of CTPM over western (chemical) drugs with similar effects in alleviating symptoms of AURI, as shown in the comparison analysis (Table 2). However, the drug costs spending on CPTM per visit was not that economical, which is almost the same with antibiotics and higher than nonantibiotic anti-infective drugs as well as other specialty medicines. It is worth noting that, by contrast, the expenditure for Chinese traditional herbal materials is exceedingly low at $3.71 \mathrm{CNY}$,being less than $10 \%$ of the CTPM cost. More clinical studies and economic evaluation concerning the efficacy, safety and costeffectiveness of the CTPM drugs are needed.

The finding also showed the overall percentage of child outpatients prescribed antibiotics was $43.8 \%$, which was moderate compared with some existing studies. It was reported to be $58.7 \%$ in Korea, $68.1 \%$ in India, $66.4 \%$ in Japan, $18.9 \%$ in Germany, and $46 \%-48 \%$ in the United States [26-32]. Compared with previous studies in China, however, vast discrepancies existed. In a systematic review and a meta-analysis[33, 34], the percentage of antibiotic prescription was both reported to be more than $80 \%$. In other regional research, it was reported that the rate of antibiotics prescribed for children with AURI was $68 \%$ in township hospitals in Guangxi Province, $5.9 \%$ in a district hospital in Beijing, $78 \%$ in 48 primary health care facilities from six 
provinces $[10,35,36]$. The possible reason underlying the large discrepancies may be that the previous studies were limited to the sample size and the specific studied facilitate or region.

It was also declared in the previous study that there was a significant reduction of antibiotic consumption after an action plan in China until 2014 [4]. However, in our study, the utilization of antibiotics related to AURI among children showed the opposite tendency between 2015-2017. The controversial results may indicate the uncertain effect of such efforts in the case of outpatient care for AURI.

In terms of the costs, the total outpatient care expenditure per encounter of uncomplicated AURI patients remained at a moderate level. But the share of out-of-pocket payment is still dominant though slightly decreased over time. Despite the tremendous progress achieved in promoting universal healthcare in the country, it indicated that the financial support for children's outpatient care is still not enough. Meanwhile, disparities could be seen in different subgroups, the health insurance type of pediatric patients had impact on prescriptions and costs. The cost disparities were likely resulted from various types and quantities of services provided to patients based on the physicians' decisions, considering financial situations of the patients and reimbursement policies of their health plans. More robust payment strategies such as prospective outpatient case-based payment for specific category of disease should be designed and control the financial uncertainty from the demand side.

This study mobilized the detail information from patient-level medical records in Chinese hospitals nationwide to describe the current situation of the prescribing pattern and spending. There are several limitations. First, the data source was from the supply side. Thus, the information on the real consumption of drugs among children before and after they left the hospitals was not obtained. The results of the study cannot describe actual utilization of medications. Second, though the selected hospitals are distributed in 31 provinces in mainland China that covered a substantial population and regions, they were not selected randomly. And we mainly focus on public hospitals and did not include the primary health care facilitates which may draw another picture. Third, the big data may superiorly overcome some challenge in regional studies and can significantly increase the statistical power of population-based studies. But it still cannot entirely represent the whole situation. Finally, there are other critical factors such as regulation policies influencing prescription practices and costs, that were not considered in our analysis. A subsequent study with factor analyses could help better understand the issues.

\section{Conclusions}

The high volume of CPTM usage is the typical feature in outpatient care of AURI pediatric patients in China. The rational and cost-effective use of CPTM and antibiotics still faces challenges. The reimbursement for child AURI cases needs to be enhanced.

\section{Abbreviations}

AURI: Acute upper respiratory tract infection; NLP: natural language processing; CPTM: Chinese traditional patent medicines; URBMI: Urban Resident Basic Medical Insurance; NRCMS: New Rural Cooperative Medical System; CHI: Commercial Health Insurance; WHC: Welfare Health Care; PHC: Preferential Health Care; OOP: 
Out-of-pocket Payment; ED: Emergency department; EC: expert consultation; GC: general consultation; EDEC: Expert consultation in the emergency department; EDGC: General consultation in the emergency department.

\section{Declarations}

\section{Ethics approval and consent to participate}

This study was approved by the Institutional Review Board of Chinese People's Liberation Army General Hospital.

\section{Consent for publication}

Not applicable

\section{Availability of data and materials}

The datasets used and/or analyzed during the current study are available from the corresponding author on reasonable request.

\section{Competing interests}

The authors declare that they have no competing interests.

\section{Funding}

The study was supported by the China National key research and development program funded by Ministry of Science and Technology of the People's Republic of China (2017YFC090840), the project funded by The General Hospital of People's Liberation Army fund (2017MBD-001) \and the program funded by National Natural Science Foundation of China (71704033).

\section{Authors' contribution}

GR, QL, SW, and LL conceived and initially designed the study. SW and LL wrote the manuscript of the report. SW, LL, NG, QL, and GR edited the manuscript. LL and XC led the data collection. SW, LL, JL, and XC provided data management. JL, SW, LL, LQM, QZ, LM, and JZ conducted data standardization. SW, LL, JL and NG did statistical analysis. LM and JZ provided medical professional support. All authors had commented on and reviewed the manuscript.

\section{Acknowledgements}

Not applicable.

\section{References}

1. China National health commission. Chinese statistical yearbook of health care 2018. Beijing: Peking Union Medical College Press; 2018. 
2. Wang Y-Y, Du P, Huang F, Li D-J, Gu J, Shen F-M, et al. Antimicrobial prescribing patterns in a large tertiary hospital in Shanghai, China. Int J Antimicrob Agents. 2016;48:666-73. https:// doi: 10.1016/j.ijantimicag.2016.09.008.

3. Li J, Song X, Yang T, Chen Y, Gong Y, Yin X, et al. A systematic review of antibiotic prescription associated with upper respiratory tract infections in China. Medicine (Baltimore). 2016;95 (19):e3587. https://doi:10.1097/MD.0000000000003587.

4. Bao L, Peng R, Wang Y, Ma R, Ren X, Meng W, et al. Significant reduction of antibiotic consumption and patients' costs after an action plan in China, 2010-2014. PloS one. 2015;10:e0118868. https:// doi: 10.1371/journal.pone.0118868.

5. Hui L, Li X-S, Zeng X-J, Dai Y-H, Foy HM. Patterns and determinants of use of antibiotics for acute respiratory tract infection in children in China. Pediatr Infect Dis J 1997;16:560-4.

6. Liu XX, Li Y, Zhu Y, et al. Seasonal pattern of influenza activity in a subtropical city, China, 2010-2015. Sci Rep. 2017;7(1):17534.

7. Yang J, Jit M, Leung KS, et al. The economic burden of influenza-associated outpatient visits and hospitalizations in China: a retrospective survey. Infect Dis Poverty. 2015;4:44.

8. Liang X, Xia T, Zhang X, Jin C. Governance structure reform and antibiotics prescription in community health centres in Shenzhen, China. Fam Pract. 2014;31(3):311-318.

9. Wang YY, Du P, Huang F, et al. Antimicrobial prescribing patterns in a large tertiary hospital in Shanghai, China. Int J Antimicrob Agents. 2016;48(6):666-673.

10. Wang J, Wang P, Wang X, Zheng Y, Xiao Y. Use and prescription of antibiotics in primary health care settings in China. JAMA Intern Med. 2014;174(12):1914-1920.

11. Mao W, Vu H, Xie Z, Chen W, Tang S. Systematic review on irrational use of medicines in China and Vietnam. PloS one. 2015;10: e0117710.

12. Wu Y, Yang C, Xi H, Zhang Y, Zhou Z, Hu Y. Prescription of antibacterial agents for acute upper respiratory tract infections in Beijing, 2010-2012. Eur J Clin Pharmacol. 2016;72(3):359-364.

13. Yin $X$, Song $F$, Gong $Y$, et al. A systematic review of antibiotic utilization in China. J Antimicrob Chemother. 2013;68(11):2445-2452.

14. Li X, Lu J, Hu S, et al. The primary health-care system in China. Lancet 2017; 390: 2584-94.

15. Li HM, Chen YC, Gao HX, et al. Effectiveness evaluation of quota payment for specific diseases under global budget: a typical provider payment system reform in rural China. BMC Health Serv. Res 2018; 18 : 635.

16. Zhang A, Nikoloski Z, Mossialos E. Does health insurance reduce out-of-pocket expenditure? Heterogeneity among China's middle-aged and elderly. Soc. Sci. Med..2017; 190: 11-9.

17. International Statistical Classification of Diseases and Related Health Problems-10th revision (Chinese edition 2012). China National Health Commission; http://www.nhc.gov.cn/mohwsbwstjxxzx/s8553/201202/54034.shtml. Accessed 5 Nov 2018.

18. Weng WH, Wagholikar KB, McCray AT, Szolovits P, Chueh HC. Medical subdomain classification of clinical notes using a machine learning-based natural language processing approach. BMC Med Inform 
Decis Mak. 2017;17(1):155.

19. Chinese Pharmacopoeia Commission: Chinese Pharmacopoeia(2015 edn.). http://eng.sfda.gov.cn/WS03/CL0757/122060.html. Accessed 5 Dec 2018.

20. National Bureau of Statistics of China: The partition of China's economic regions. http://www.stats.gov.cn/ztjc/zthd/sjtjr/dejtjkfr/tjkp/201106/t2011061371947.htm. Accessed 1 Dec 2018.

21. Lin W, Liu GG, Chen G. The Urban Resident Basic Medical Insurance: a landmark reform towards universal coverage in China. Health Econ. 2009;18 Suppl 2:S83-96.

22. Medication use for pediatric upper respiratory tract infections. Fundam Clin Pharmacol. 2006 Aug;20(4):385-90.

23. Pandey AA, Thakre SB, Bhatkule PR. Prescription analysis of pediatric outpatient practice in nagpur city. Indian J Community Med. 2010;35(1):70-73. doi:10.4103/0970-0218.62564

24. Maltezou HC, Dedoukou X, Asimaki H, et al. Consumption of antibiotics by children in Greece: a crosssectional study. Int J Pediatr Adolesc Med. 2017;4(3):108-111.

25. Aabenhus R, Hansen MP, Saust LT, Bjerrum L. Characterisation of antibiotic prescriptions for acute respiratory tract infections in Danish general practice: a retrospective registry based cohort study. NPJ Prim Care Respir Med. 2017;27(1):37.

26. Shin SM, Shin J-Y, Kim MH, Lee SH, Choi S, Park B-J. Prevalence of antibiotic use for pediatric acute upper respiratory tract infections in Korea. J Korean Med Sci. 2015;30:617-24. https:// doi: 10.3346/jkms.2015.30.5.617.

27. Majhi B, Panda A, Barma SK. Antibiotic prescribing pattern in paediatrics outpatient in a tertiary care hospital. J Evid Based Med. 2017;4:3048-51. https:// DOI: 10.18410/jebmh/2017/604.

28. Yoshida S, Takeuchi M, Kawakami K. Prescription of antibiotics to pre-school children from 2005 to 2014 in Japan: a retrospective claims database study. J Public Health (Oxf). 2017;40:397-403.https:// doi: 10.1093/pubmed/fdx045.

29. Schindler C, Krappweis J, Morgenstern I, Kirch W. Prescriptions of systemic antibiotics for children in Germany aged between 0 and 6 years. Pharmacoepidemiol Drug Saf. 2003;12:113-20. https:// doi: 10.1002/pds.786.

30. Nyquist A-C, Gonzales R, Steiner JF, Sande MA. Antibiotic prescribing for children with colds, upper respiratory tract infections, and bronchitis. JAMA. 1998;279:875-7.

31. Agiro A, Gautam S, Wall E, Hackell J, Helm M, Barron J, et al. Variation in Outpatient Antibiotic Dispensing for Respiratory Infections in Children by Clinician Specialty and Treatment Setting. Pediatr Infect Dis J. 2018;37:1248-54.https:// doi: 10.1097/INF.0000000000002004.

32. Fleming-Dutra KE, Hersh AL, Shapiro DJ, et al. Prevalence of Inappropriate Antibiotic Prescriptions Among US Ambulatory Care Visits, 2010-2011. JAMA. 2016;315(17):1864-1873.

33. Li J, Song X, Yang T, Chen Y, Gong Y, Yin X, et al. A systematic review of antibiotic prescription associated with upper respiratory tract infections in China. Medicine (Baltimore). 2016;95 (19):e3587. https://doi:10.1097/MD.0000000000003587. 
34. Li WM, Lu YL, Chen MY, Yin G, Zeng XY. Usage of antibiotics among children with upper respiratory tract infections in China before and after the new health care reforms: Meta Analysis. Chinese Pharmaceutical Journal. 2017;52(10):880-5(in Chinese).

35. Zhang Z, Hu Y, Zou G, Lin M, Zeng J, Deng S, et al. Antibiotic prescribing for upper respiratory infections among children in rural China: a cross-sectional study of outpatient prescriptions. Glob Health Action. 2017;10:1287334. https://doi: 10.1080/16549716.2017.1287334.

36. Hu XL, Zhang ZQ. Investigation of antibiotic prescriptions among children with upper respiratory tract infections. Chinese General Practice. 2016;1(in Chinese).

37. Wang J, Wang P, Wang $X$, Zheng Y, Xiao Y. Use and prescription of antibiotics in primary health care settings in China. JAMA Intern Med.2014; 174:1914-20. https:// doi:

10.1001/jamainternmed.2014.5214.

\section{Figures}
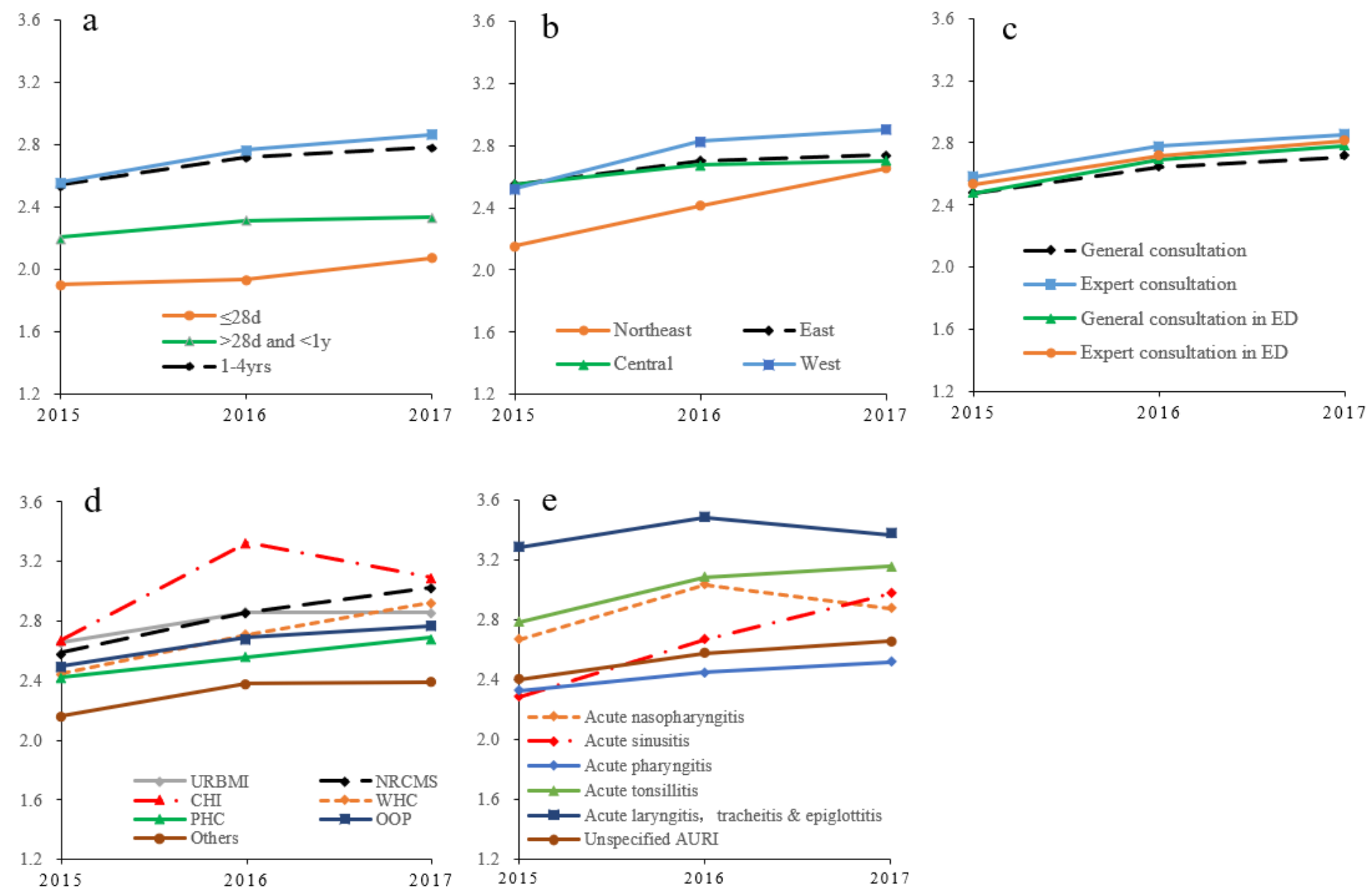

Figure 1 
Comparison of average numbers of drugs prescribed per encounter among subgroups in 2015-2017. a: ages. b: economic regions. c: consultation types. d: health insurance and health care plan types. e: AURI classifications. X-axis: median costs per visit (CNY), Y-axis: year.

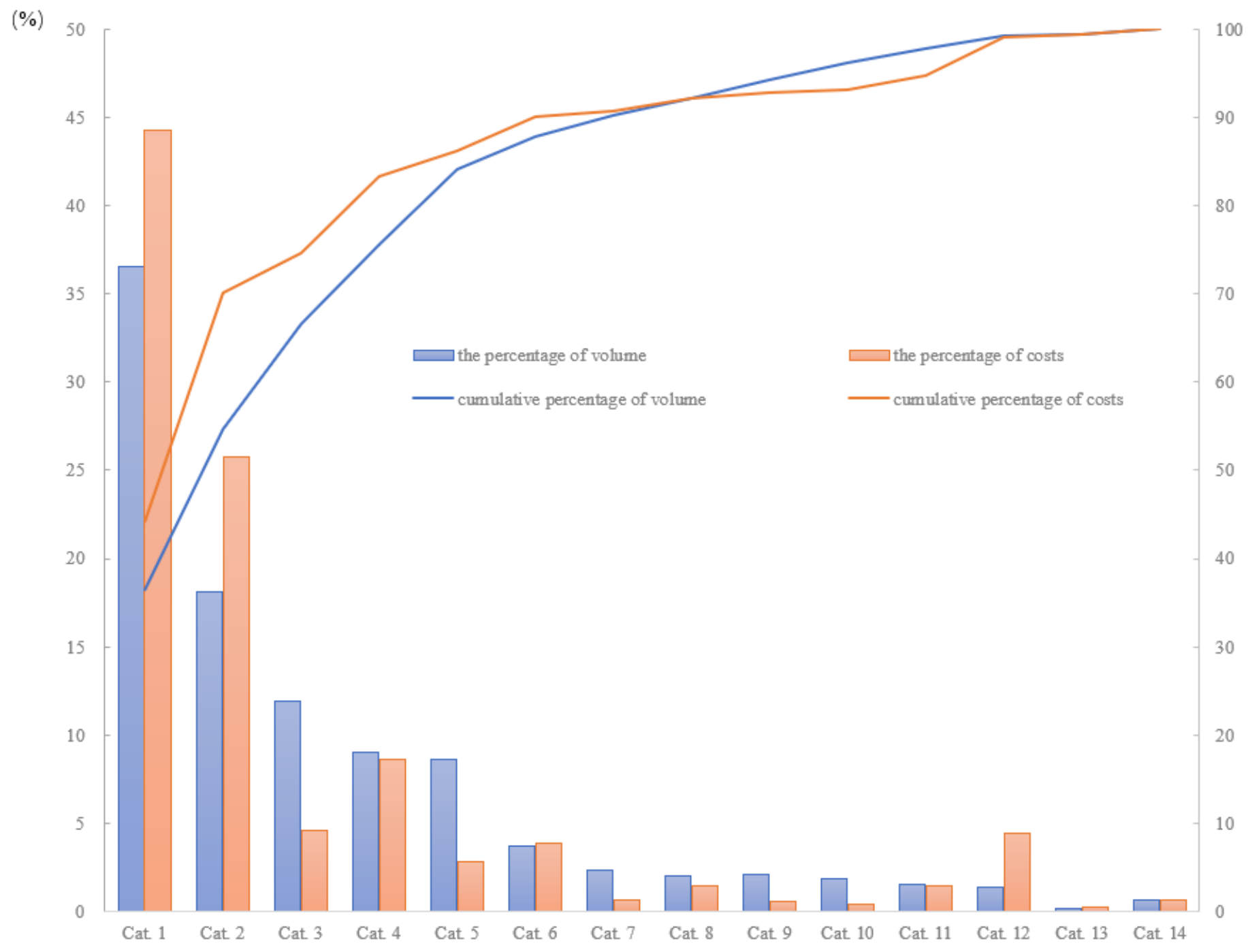

\section{Figure 2}

The percentages and cumulative percentages of prescription frequencies and costs of drugs. Cat. 1=Chinese traditional patent medicine Cat. 2=Antibiotic anti-infective drugs Cat. $3=$ Antipyretic-analgesics drugs Cat. 4=Respiratory system drugs Cat. $5=$ Water, electrolyte $\&$ acid-base balance regulating drugs Cat. $6=$ Nonantibiotic anti-infective drugs Cat. 7=Antiallergic drugs Cat. 8=Digestive system drugs Cat. 9=Vitamins \& minerals Cat. 10=Hormones \& endocrine regulating drugs Cat. 11=Specialist drugs Cat. $12=$ Immunoregulation drugs Cat. 13=Traditional Chinese medicinal materials Cat. 14=0thers 
a

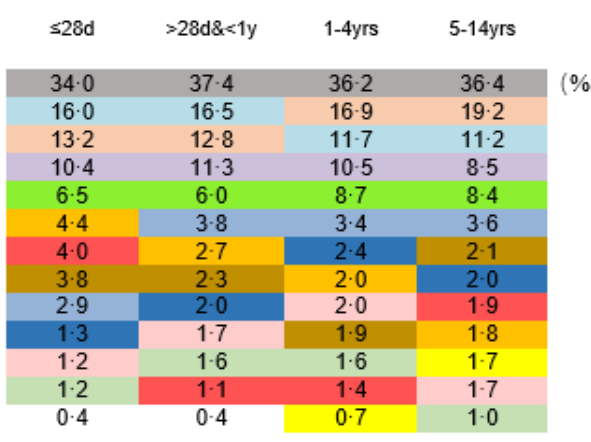

b

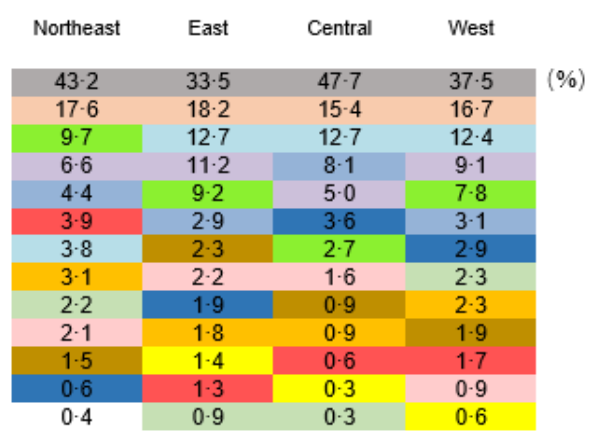

d

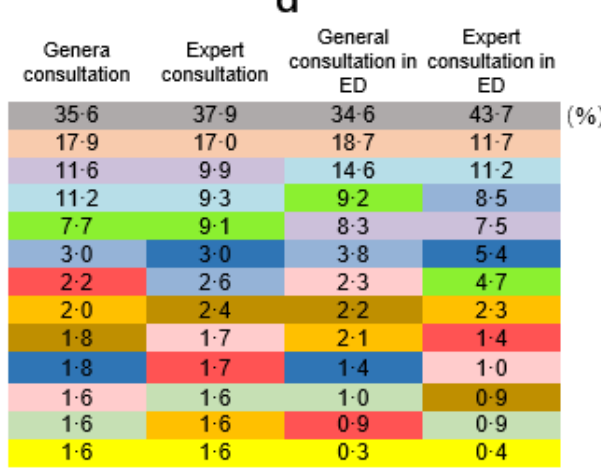

\begin{tabular}{|c|c|c|c|c|c|c|}
\hline URBMI & NRCMS & $\mathrm{CHI}$ & WHC & $\mathrm{PHC}$ & OOP & Others \\
\hline $36 \cdot 4$ & $35 \cdot 0$ & $38-8$ & $39 \cdot 2$ & $29 \cdot 5$ & $36 \cdot 0$ & $47-7$ \\
\hline $18 \cdot 2$ & $14 \cdot 6$ & $17-1$ & $17 \cdot 6$ & $15 \cdot 5$ & $17 \cdot 4$ & $16-3$ \\
\hline $12 \cdot 0$ & $12 \cdot 6$ & $10 \cdot 7$ & $15 \cdot 9$ & $12 \cdot 9$ & $11 \cdot 1$ & 9.5 \\
\hline $11 \cdot 6$ & $12 \cdot 1$ & $9 \cdot 1$ & $10 \cdot 9$ & 11.4 & $9 \cdot 7$ & $6 \cdot 9$ \\
\hline $6 \cdot 8$ & $8-2$ & $7 \cdot 4$ & $3 \cdot 7$ & $10 \cdot 1$ & $9 \cdot 1$ & $6 \cdot 0$ \\
\hline $2 \cdot 9$ & $3 \cdot 8$ & $2 \cdot 9$ & $2 \cdot 9$ & $7 \cdot 6$ & 3.9 & $2 \cdot 8$ \\
\hline $2 \cdot 5$ & $3 \cdot 4$ & $2 \cdot 6$ & $2 \cdot 0$ & 3.4 & $2 \cdot 3$ & $2 \cdot 6$ \\
\hline $2 \cdot 1$ & $3 \cdot 2$ & $2 \cdot 6$ & 1.8 & $3 \cdot 3$ & $2 \cdot 2$ & $1 \cdot 7$ \\
\hline $1 \cdot 7$ & $1 \cdot 8$ & $2 \cdot 5$ & 1.8 & $2 \cdot 0$ & $2 \cdot 2$ & $1 \cdot 4$ \\
\hline $1 \cdot 6$ & $1 \cdot 8$ & $1 \cdot 6$ & $1-4$ & 1.5 & 1.9 & $1 \cdot 4$ \\
\hline $1 \cdot 4$ & $1 \cdot 0$ & $1 \cdot 4$ & $1 \cdot 1$ & $1 \cdot 2$ & $1 \cdot 6$ & $1 \cdot 3$ \\
\hline $1 \cdot 2$ & $1 \cdot 0$ & $1 \cdot 1$ & 0.9 & 0.7 & 1.5 & $1 \cdot 0$ \\
\hline $1 \cdot 2$ & 0.6 & $1 \cdot 0$ & 0.5 & 0.7 & 0.6 & 0.7 \\
\hline
\end{tabular}

\section{Figure 3}

Prescription Diversity among subgroups. a: ages. b: economic regions. c: consultation types. d: health insurance and health care plan types. e: AURI classifications. The colored squares represent different major categories of drugs; the number in the square shows the percentage of the major category of drugs prescribed in its subgroup. 

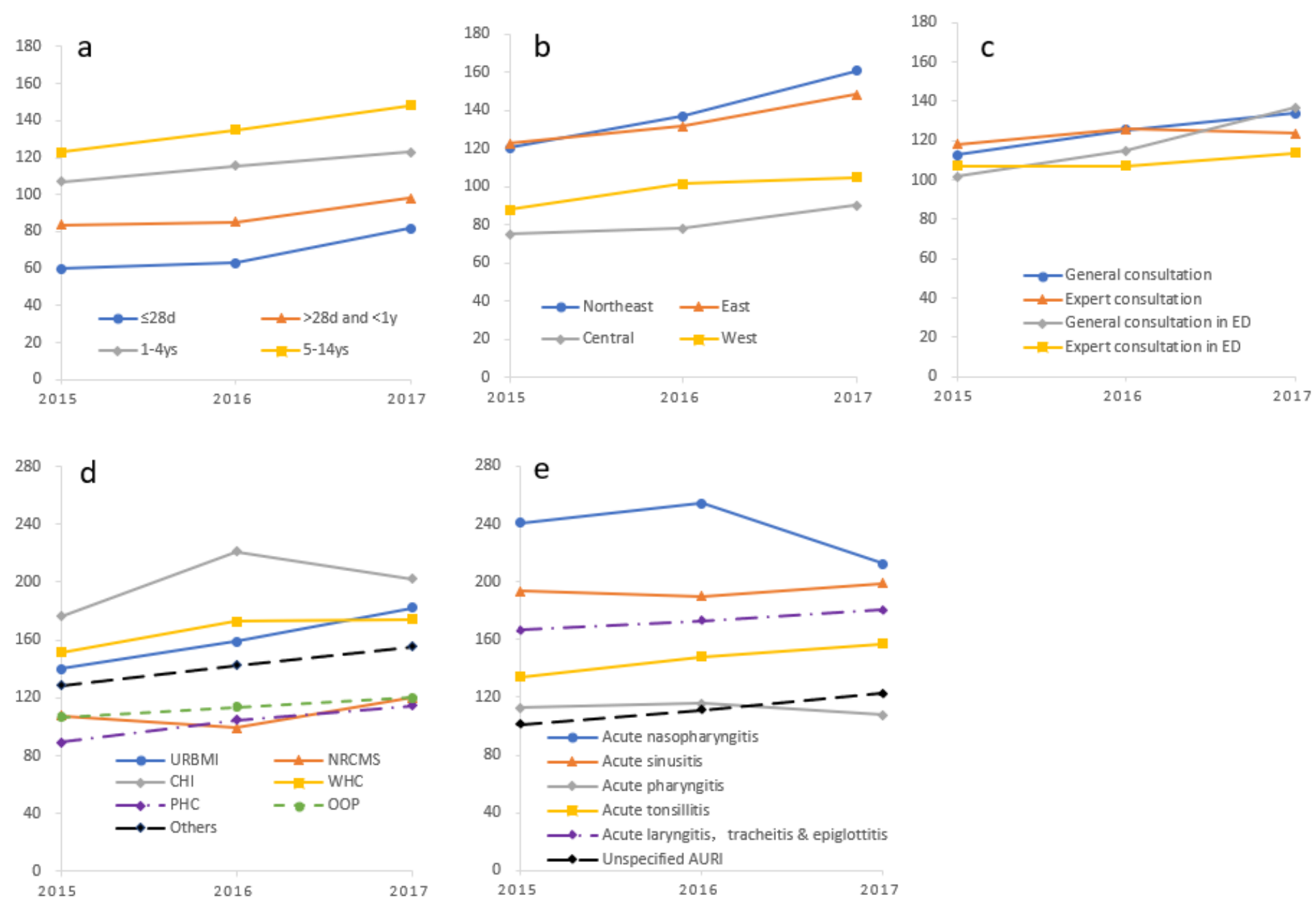

\section{Figure 4}

Comparison of median costs per visit among subgroups in 2015-2017. a: ages. b: economic regions. c: consultation types. d: health insurance and health care plan types. e: AURI classifications. X-axis: median costs per visit (CNY), Y-axis: year. 